\title{
Diagnostik und Therapie schlafbezogener Atmungsstörungen im Zusammenhang mit der Corona-Pandemie*
}

\author{
Deutsche Gesellschaft für Pneumologie und Beatmungsmedizin e. V. (DGP) \\ Deutsche Gesellschaft für Schlafforschung und Schlafmedizin (DGSM)
}

\section{Management of Diagnostic Procedures and Treatment of Sleep Related Breathing Disorders in the Context of the Coronavirus Pandemic}

\author{
German Respiratory Society (DGP) \\ German Sleep Society (DGSM)
}

N. Büchner ${ }^{1}$, H. Woehrle' ${ }^{2}$ D. Dellweg ${ }^{3}$, A. Wiater ${ }^{4}$, P. Young ${ }^{5}$, H. Hein ${ }^{6}$, W. Randerath ${ }^{7,8}$

\author{
Autoren \\ Institute \\ 1 Medizinische Klinik I (Pneumologie, Schlaf- und \\ Beatmungsmedizin), Helios Klinikum Duisburg GmbH, \\ Duisburg \\ 2 Lungenzentrum Ulm \\ 3 Fachkrankenhaus Kloster Grafschaft GmbH, \\ Akademisches Lehrkrankenhaus der Philipps-Universität \\ Marburg, Schmallenberg Grafschaft \\ 4 Deutsche Gesellschaft für Schlafforschung und \\ Schlafmedizin, Schwalmstadt-Treysa \\ 5 Medical Park, Neurologische Klinik Reithofpark, Bad \\ Feilnbach \\ 6 Praxis und Schlaflabor für Innere Medizin, Pneumologie, \\ Allergologie, Schlafmedizin, Reinbek \\ 7 Klinik für Pneumologie, Krankenhaus Bethanien, \\ Solingen \\ 8 Institut für Pneumologie an der Universität zu Köln, Köln
}

Bibliografie

DOI https://doi.org/10.1055/a-1184-8442 |

Online-Publikation: 10.6 .2020 |

Pneumologie 2020; 74: 571-581

(c) Georg Thieme Verlag KG Stuttgart · New York ISSN 0934-8387

Korrespondenzadresse

PD Dr. med. Nikolaus Büchner, Medizinische Klinik I

(Pneumologie, Schlaf- und Beatmungsmedizin),

Helios Klinikum Duisburg GmbH,

An der Abtei 7-11, 47166 Duisburg

nikolaus.buechner@rub.de

\section{Einleitung}

Ausgehend von der Millionenstadt Wuhan in der chinesischen Provinz Hubei entwickelte sich im Januar 2020 durch das bis dahin unbekannte Corona-Virus SARS-CoV-2 in China eine Epidemie, die sich mittlerweile weltweit zur Corona-Pandemie entwickelt hat. In Deutschland konnte bislang in den meisten Regionen eine Überlastung des Gesundheitssystems, wie sie in einigen europäischen Ländern und auch weltweit zu beobachten war, vermieden werden. Regional findet sich jedoch ein deutlich unterschiedliches Auftreten von Corona-Fällen, wobei allgemein ein Süd-Nord- und West-Ost-Gefälle zu beobachten ist. Dies ist einerseits durch die Nähe zu ehemaligen Risiko-

\footnotetext{
* Dieser Beitrag erscheint zeitgleich in den Zeitschriften Pneumologie und Somnologie.
}

gebieten, andererseits durch lokale Ausbrüche (z. B. Landkreis Heinsberg) zu erklären.

Die Übertragung in der Bevölkerung erfolgt vorwiegend aerogen durch Tröpfcheninfektion [3,4], möglicherweise aber auch durch Aerosole insbesondere im medizinischen Umfeld bei Interventionen in der Nähe der Atemwege (Bronchoskopie, Intubation, Maskenbeatmung), wahrscheinlich aber auch im normalen gesellschaftlichen Umgang (RKI-Steckbrief zu COVID19, Übertragungswege, 7. Mai 2020). Eine relevante Infektiosität besteht bereits $2-3$ Tage vor Symptombeginn [5], die Dauer der Ansteckungsfähigkeit ist unklar, die höchste Infektiosität fand sich am Tag vor dem Symptombeginn [6]. Tatsächlich wird der Anteil einer prä- oder asymptomatischen Übertragung zwischen 44 und $62 \%$ geschätzt [6,7]. Zu diesem Zeitpunkt zeigt sich eine hohe Rate falsch-negativer Befunde beim PCR-Direktnachweis von SARS-CoV-2-Viren [8]. 
Zu den klinisch relevanten Symptomen zählen Geruchs-/Geschmacksstörungen, Fieber, Husten, Halsschmerzen, allgemeine Schwäche, Schmerzen, laufende Nase und Durchfall. Die Krankheitsverläufe gestalten sich jedoch sehr variabel von asymptomatischen Verläufen bei $22-43 \%$ [9-11] bis hin zu schweren Pneumonien mit Lungenversagen und Tod.

Die Rate schwerer und kritischer Verläufe, die Hospitalisierungs- und Sterblichkeitsraten lassen sich immer noch nicht zuverlässig angeben, u. a. da der Anteil asymptomatisch verlaufender Infektionen in der Bevölkerung immer noch nicht flächendeckend valide untersucht wurde. Außerdem besteht eine Heterogenität bei der Erkennung und Registrierung von positiven Fällen, was den Vergleich entsprechender Kenngrößen (z.B. case fatality proportion $=$ Todesfälle/Gesamtzahl registrierter Fälle) erschwert. Nach einer Modellrechnung in Frankreich [1214] beträgt die Hospitalisierungsrate unter den Infizierten $2,6 \%$ und die Sterblichkeitsrate der Infektion (sog. Infektionssterblichkeit, infection fatality rate, IFR) unter allen SARS-CoV2-Infizierten wahrscheinlich unter $1 \%$, in Frankreich kalkuliert wurden 0,53\% mit einer Zunahme bei höherem Alter (<20 Jahre: 0,001\%, > 80 Jahre: 8,3\% [95\% KI: 4,4 - 13,9]). Nach Erhebungen aus Gangelt (12597 Einwohner) im nordrheinwestfälischen Landkreis Heinsberg (Hotspot) 6 Wochen nach dem dortigen Ausbruch waren 15,5\% (95\% KI: 12,3-19,0\%) mit dem SARSCoV-2-Virus infiziert, was einer 5-fach höheren Rate als die offiziell gemeldeten Fälle $(3,1 \%)$ entsprach. Bezogen auf die so hochgerechnete Zahl der in der Bevölkerung Infizierten lag die IFR in Heinsberg bei 0,36\% (95\% KI: 0,29-0,45\%) [10] und nach einer Stichprobe $(n=3,300)$ in Santa Clara County in Kalifornien bei 0,17\% [15]. Zum Zeitpunkt der Manuskripterstellung beträgt bei COVID-19 die Todesrate bezogen auf 100000 Einwohner in Deutschland 8. Sie liegt damit deutlich unterhalb der in anderen europäischen Ländern beobachteten [16].

Zu den Risikofaktoren für einen schweren Verlauf zählen gemäß RKI höheres Alter (stetige Zunahme ab 50-60 Jahren), Nikotinabusus, schweres Übergewicht, kardiovaskuläre, chronische pulmonale (z. B. COPD) und hepatische Komorbiditäten, Diabetes mellitus und Krebserkrankungen sowie ein krankheitsoder medikamentös bedingt geschwächtes Immunsystem [17, 18].

Zwar entwickelt sich die Zahl der Neuinfektionen momentan linear, nach und nach sehen wir aber vermehrt Ausbrüche bei Patienten mit erhöhtem Risiko in Pflegeheimen, Rehakliniken und in Akutkliniken. Die Entwicklung der Neuinfektionen nach erster Lockerung der Maßnahmen zur Kontaktreduktion ab dem 06.05. 2020 bleibt abzuwarten.

Zu Beginn der Corona-Krise wurde auf Aufforderung des Bundesministeriums für Gesundheit aber auch durch Unsicherheit seitens der Patienten eine Schließung von Schlaflaboren als vermeintlich elektive Versorgungseinheit vielerorts erzwungen. Außerdem verhinderten die staatlich verordnete Kontaktsperre sowie lokale Vorgaben der Infektprävention bislang oft den Normalbetrieb im Schlaflabor. Hinzu kommt, dass zumindest im Krankenhaus Schlaflaborpersonal teilweise umorganisiert/umgeschult wurde und Räumlichkeiten und ggf. medizinisches Equipment für die Akutversorgung von Corona-Patienten reserviert wurden.
Unter dem Einfluss der Corona-Pandemie kam es auf diese Weise europaweit in den ersten 2 Monaten zu einer erheblichen Einschränkung schlafmedizinischer Leistungen. Eine entsprechende Erhebung zur Änderung der Diagnostik und Therapie in fast ausschließlich stationären Schlaflaboren zeigte einen starken Rückgang der PSG im Schlaflabor von 92,5 auf $20 \%$, ebenso fand sich ein Rückgang häuslicher diagnostischer Polygrafien von 87,5 auf $32,5 \%$. Die Nutzung telemedizinischer Verfahren blieb in etwa gleich (30,0 vs. 27,5\%). Zudem nahm der Anteil von Schlaflaboren, die Therapieanpassungen und -kontrollen durchführten, auf 17,5\% bzw. 7,5\% ab [19].

Aufgrund eines bislang nicht verfügbaren Impfstoffes und einer nur langsam erfolgenden Durchseuchung der Bevölkerung ist das Ende der Corona-Pandemie nicht zuverlässig vorhersagbar. Daher ist es erforderlich, zumindest mittelfristig tragbare Strukturen auch in der schlafmedizinischen Versorgung unter dem Einfluss der andauernden Corona-Pandemie und darüber hinaus zu definieren.

In Bezug auf nichtinvasive Beatmungsverfahren bei nachgewiesener COVD-19 liegt mittlerweile ein Positionspapier der DGP vor [1]. Konkrete verbindliche Vorgaben zur Durchführung schlafmedizinischer Diagnostik im ambulanten und stationären Sektor bei Patienten mit unbekanntem SARS-CoV-2-Infektionsstatus fehlen in Deutschland bislang. Ebenso liegen keine Empfehlungen zum Umgang mit PAP-Verfahren in Hinblick auf das Risiko durch Aerosolbildung im häuslichen Bereich bei möglicher COVID-19-Erkrankung vor. Ferner bestehen Bedenken auf Seiten des Schlaflaborpersonals bezüglich eines erhöhten Infektionsrisikos durch vermehrte Aerosolbildung bei unentdeckter SARS-CoV-2-Infektion im Rahmen der Anpassung oder Kontrolle einer nächtlichen Überdrucktherapie bei vorwiegendem Einsatz von Leckage-Masken.

Naturgemäß liegen zu diesen Fragestellungen noch keine großen Erfahrungen und wissenschaftlich fundierte Erkenntnisse vor, somit basieren viele Stellungnahmen und Empfehlungen auf klinischen und epidemiologischen Beobachtungen, Modellen und Vorhersagen.

In dieser Situation besteht die Gefahr, dass durch vorübergehende Schließung stationärer und auch ambulanter Schlaflabore andere, nicht leitlinienbasierte Diagnostik- und Therapiewege etabliert werden, wie z. B. die Therapieeinleitung ohne vorherige apparative Diagnostik zur Schweregradbestimmung und genauen Differenzierung einer SBAS. Dies ist zwar abzulehnen, aber dennoch erscheint es geboten, unter dem Einfluss der Beschränkungen im Rahmen der Corona-Pandemie neue Versorgungsstrukturen unter Einhaltung evidenzmedizinisch begründeter und leitlinienbasierter Standards kritisch zu diskutieren.

Dieses Manuskript knüpft an die Empfehlungen aus dem Positionspapier der DGP zur praktischen Umsetzung der apparativen Differenzialtherapie der akuten respiratorischen Insuffizienz bei COVID-19 an und versteht sich wie dieses als Ergänzung, Fortführung und Impulsgeber für einen fachübergreifenden Konsens, basierend auf bestehenden Leitlinien [2].

Für die Schlafmedizin ergeben sich neben den apparativen Gemeinsamkeiten und Überschneidungen zur Beatmungsmedizin noch zusätzliche Fragestellungen. 
- So ist bislang im Regelfall bei Patienten zum Zeitpunkt der schafmedizinischen Untersuchung nicht bekannt, ob sie mit SARS-CoV-2 infiziert sind.

- Aufgrund der Dunkelziffer nicht-erfasster Corona-Fälle muss aufgrund des hohen Patientenumsatzes trotz aller vorbeugender Maßnahmen mit SARS-CoV-2-Kontakten im Schlaflabor gerechnet werden.

- Patienten mit Schlafapnoe haben oft Risikofaktoren für einen schweren Verlauf einer COVID-19 und sollten daher besonderen Schutz vor einer Infektion im Schlaflabor erhalten.

- Außerdem stellt sich ferner die Frage, wie das Risiko einer Ausbreitung und auch Gefährdung des medizinischen Personals zu bewerten ist und unter welchen Bedingungen schlafmedizinische Leistungen überhaupt (wieder) angeboten werden können.

Die Schlafapnoe selbst erhöht wahrscheinlich nicht das Risiko für eine SARS-CoV-2-Infektion (Statement der DGSM 22.04. 2020). Allerdings sind Schlafapnoe-Patienten oft durch Begleiterkrankungen in einer höheren Risikogruppe für einen schweren COVID-19-Verlauf einzustufen [17], auch eine Verschlechterung der COVID-19 z.B. durch chronische proinflammatorische Prozesse bei OSA [20] wäre denkbar, insbesondere bei gleichzeitig bestehender Adipositas [21]. In einer vergleichenden Darstellung von 50 hospitalisierten COVID-19-Fällen mit $(n=24)$ und ohne $(n=26)$ ARDS zeigte sich kein Unterschied hinsichtlich der Häufigkeit vorbekannter Schlafapnoe $(n=2 / 24$ [8\%] vs. $n=5 / 26$ [19\%]), dies jedoch nur nach Sichtung der Vorbefunde und ohne systematische Diagnostik hinsichtlich einer SBAS [18].

Durch Konzentrierung medizinischer Leistungen (Personal, Material, räumliche Ressourcen) zur Sicherstellung einer adäquaten Notfallversorgung eines erwarteten hohen COVID-19Aufkommens und Furcht von Patienten, eine medizinische Einrichtung aufzusuchen, kommt es vielerorts zu einer Unterversorgung in anderen medizinischen Bereichen. Der resultierende gesundheitliche Schaden durch eine Unterversorgung chronischer Erkrankung, aber auch in der Notfallmedizin von nichtCOVID-19-assoziierten Notfällen ist bislang nicht absehbar.

Aufgrund sozialer (Unfallrisiko, Berufsfähigkeit) und prognostischer (z.B. kardiovaskuläre Komplikationen) Faktoren kann eine Verzögerung bei Diagnostik und Therapieeinleitung einer schlafbezogenen Atmungsstörung nicht länger verantwortet werden. Der Einfluss der momentanen Unterversorgung in der Schlafmedizin durch verzögerte Diagnosestellung und Therapieeinleitung ist ebenfalls noch schwer einschätzbar. Allerdings führt schon eine kurzzeitige Unterbrechung der CPAP-Therapie zu einer teilweise deutlichen kardiovaskulären Belastung [22], und unfallbezogene Risiken im Straßenverkehr, aber auch im beruflichen Umfeld nehmen zu. Im Umkehrschluss ist daher auch eine kurze Therapieverzögerung nicht bedenkenlos zu akzeptieren. In diesem Sinne versteht sich die schlafmedizinische Versorgung nicht als eine rein elektive und daher länger verzichtbare oder verschiebbare Leistung, sondern als eine in vielen Fällen dringliche medizinische Versorgung. Eine Fortfüh- rung bzw. Wiederaufnahme der schlafmedizinischen Versorgung ist daher dringend anzustreben.

Wegen des stetigen Erkenntniszuwachses kann die aktuelle Stellungnahme nur eine Momentaufnahme sein, basierend auf der aktuellen Entwicklung, der aktuellen Ausbreitungslage und den jeweiligen aktuellen Empfehlungen z.B. des Robert-KochInstitutes.

\section{Maßnahmen für den Betrieb eines Schlaf- labores bzw. Durchführung schlafmedizini- scher Leistungen}

\section{Allgemeine Hinweise und Organisation}

Grundsätzlich sind aktuelle bundes- und länderspezifischen Vorgaben zu beachten. Eine Rücksprache mit dem Krankenhaushygieniker bzw. dem lokalen Gesundheitsamt ist bei Wiederaufnahme des Schlaflaborbetriebes und relevanten organisatorischen Änderungen notwendig.

Die jeweils gültigen Regeln für den Umgang im öffentlichen Leben bilden die Basis für die Empfehlungen in der medizinischen Versorgung, allerdings stellt der Praxis- und Krankenhausbetrieb besondere Anforderungen an den Schutz der Patienten und des medizinischen Personals.

Grundsätzlich ist nach wie vor auf eine konsequente Einhaltung der auch in der Öffentlichkeit geltenden Abstandsregeln (1,5-2 m) wie auch auf die Maskenpflicht zu achten. Evtl. sollte die Einrichtung einer Barriere, z. B. durch eine Plexiglaswand bei Anmeldung und Erstkontakt, erwogen werden.

Empfohlen wird unter Berücksichtigung der räumlichen Gegebenheiten eine konsequente räumliche Abtrennung des Schlaflabores, sofern im oder am Krankenhaus befindlich, in eine „Non-COVID-Zone“ mit Vermeidung von Patientenkontakt durch getrennte Zugänge und Wartebereiche.

Vor Aufnahme sollte eine angemessene Evaluation hinsichtlich des Gesundheitszustandes und des Infektions- und Erkrankungsrisikos der Patienten erfolgen, z.B. sollte 1-2 Tage vor Aufnahme (telefonisch) eine Abfrage bzgl. des Gesundheitszustandes und möglicher SARS-CoV-2-Kontakte durchgeführt werden. Bei Hinweisen für eine SARS-CoV-2-Infektion bzw. COVID-19 sollte eine Verschiebung des Termins erfolgen. Bei Aufnahme müssen die Patienten bezüglich typischer COVIDSymptome und möglicher SARS-CoV-2-Kontakte befragt werden, eine entsprechende Dokumentation wird dringend angeraten. Ferner sollte zur Vermeidung unnötiger Kontakte z. B. eine zeitlich gestaffelte Einbestellung der Patienten erfolgen sowie ggf. eine Verringerung der Kontaktzeiten durch abendliche Aufnahme.

Die in der aktuellen S3-Leitlinie „Nicht erholsamer Schlaf/ Schlafstörungen - Kapitel Schlafbezogene Atmungsstörungen“ ([2] derzeit in Überarbeitung) empfohlenen Grundlagen zur Durchführung und Überwachung einer schlafmedizinischen Diagnostik und Therapieeinleitung sind auch unter dem Einfluss der Corona-Pandemie zu berücksichtigen. Eine Leistungserbringung außerhalb dieser Vorgaben ist auch mit Verweis auf die Pandemie aus qualitativen und abrechnungstechnischen 
Aspekten abzulehnen. Dies gilt ausdrücklich auch für Geräteanpassung und Maskenanpassung durch die Provider.

\section{Priorisierung}

- Zum ersten Patientenkontakt sollte, falls vorhanden, ggf. eine Nutzung telefonischer/videotelefonischer Vorabberatungen erfolgen mit Einstufung der Notwendigkeit einer persönlichen Vorstellung zur Diagnostik, Therapieeinleitung oder -kontrolle.

- Bei unzureichenden Schlaflaborressourcen sollte eine Priorisierung bzgl. der Dringlichkeit (kardiovaskuläre und andere Komorbiditäten, Risikoberufe, Leidensdruck, soziale Faktoren wie Erhalt der Erwerbsfähigkeit bei Berufskraftfahrern etc.) vor Aufnahme in ein Schlaflabor bzw. ambulanter persönlicher Vorstellung erfolgen. Hierbei muss allerdings die allgemeine Risikolage in Bezug auf die Corona-Pandemie gegen das Risiko einer unbehandelten schlafbezogenen Atmungsstörung (kardiovaskuläre Komplikationen, Unfallrisiko) abgewogen werden. Bei zurückgestellter Diagnostik, Therapieeinleitung oder -kontrolle eines unter Therapie noch symptomatischen Patienten sollte eine Aufklärung bzgl. der resultierenden Risiken inkl. einer ggf. nicht vorliegenden medizinischen Fahrtauglichkeit erfolgen.

\section{Infektprävention im Schlaflabor}

- Maßnahmen am Patienten sollten grundsätzlich unter strikter Beachtung der Basishygiene erfolgen mit geeigneter persönlicher Schutzausrüstung (PSA, bei normalem Patientenkontakt inkl. Mund-Nasenschutz [MNS], bei engem Kontakt durch Verkabelung und Maskenanpassung/-optimierung inkl. Augenschutz und FFP2/FFP3-Maske).

- Grundsätzlich sollte nur eine Unterbringung im Einzelzimmer vorzugsweise mit eigener Nasszelle erfolgen, was ohnehin in den meisten Schlaflaboren bereits umgesetzt ist. Ein (gemeinsamer) Aufenthalt in Aufenthaltsräumen sollte nicht stattfinden und auch sonst ein Kontakt der Patienten untereinander vermieden werden.

- Besucherregelungen sollten strikt unter Berücksichtigung lokaler Vorgaben erfolgen.

- Sofern verfügbar sollte bevorzugt Einmalmaterial verwendet werden. Ansonsten ist die strikte Einhaltung der RKI- bzw. Herstellerempfehlungen zur Desinfektion und Hygiene von Oberflächen, Kabeln, PAP- und Beatmungsgeräten, Masken, Schläuchen, Filtern zu fordern.

- Auf eine gute Durchlüftung der Räume nach außen sollte geachtet werden und keine zentrale Klimaanlage zur Anwendung kommen. Raumbezogene Klimaanlagen mit Abluft nach außen sind hingegen eher unproblematisch.

- Während des Aufenthaltes sollten Kontaktzeiten im Untersuchungsraum möglichst kurz gehalten werden, z. B. durch die bevorzugte Nutzung der Sprechanlage.

\section{Screening und Schutz von Patienten und Personal}

- Bzgl. der Kriterien für eine Testung von Schlaflaborpatienten auf SARS-CoV-2-Infektion gelten die aktuellen Empfehlungen des RKI bzw. der Bundes- oder Landesbehörden. Zum Zeitpunkt der Manuskripterstellung gilt: Bei akuten respira- torischen Symptomen jeder Schwere und/oder Geruchs-/ Geschmacksstörungen, SARS-CoV-2-Kontakt innerhalb von 14 Tagen oder klinischen bzw. radiologischen Hinweisen auf eine virale Pneumonie sollte eine Testung auf SARS-CoV-2 durchgeführt werden. Bis zum Eingang des Ergebnisses sollte dann keine Untersuchung im Schlaflabor erfolgen.

- Bei Auftreten typischer Symptome oder bestehendem Kontakt mit SARS-CoV-2-Infizierten während des Aufenthaltes im Schlaflabor sollte eine Abklärung der Patienten und des medizinischen Personals gemäß der aktuellen Empfehlung des RKI erfolgen (COVID-19-Verdacht: Testkriterien und Maßnahmen. Orientierungshilfe für Ärztinnen und Ärzte, Version 12. Mai 2020, bitte Aktualisierung beachten (RKI - Allgemeine Quellen).

- Bei Symptomen oder sonstigem Verdacht auf COVID-19 (typisches Röntgen, Corona-Kontakt) und notwendiger stationärer Aufnahme außerhalb des Schlaflabores sollten Patienten mit schlafbezogenen Atmungsstörungen ihr PAPGerät mitbringen und gemäß einer auf ihren aktuellen Gesundheitszustand bezogenen ärztlichen Anordnung nutzen.

- Patienten mit Fieber oder Atemwegssymptomen sollten nicht im Schlaflabor untersucht oder behandelt werden ohne vorherige Diagnostik auf eine SARS-CoV-2-Infektion. Bei Auftreten typischer Symptome oder bestehendem Kontakt mit SARS-CoV-2-Infizierten sollten Patienten und auch das medizinische Personal gemäß der aktuellen Empfehlung des RKI (COVID-19-Verdacht: Testkriterien und Maßnahmen. Orientierungshilfe für Ärztinnen und Ärzte, Version 12. Mai 2020, bitte Aktualisierung beachten (RKI - Allgemeine Quellen) weiter abgeklärt und bis zum Eingang des Testergebnisses ambulant mit häuslicher Quarantäne bzw. stationär isoliert werden.

- Patienten, die mit Symptomen oder sonstigem Verdacht (typisches Röntgen, Corona-Kontakt) auf COVID-19 außerhalb des Schlaflabores stationär aufgenommen werden, sollten ihr CPAP-Gerät mitbringen und gemäß einer auf ihren aktuellen Gesundheitszustand bezogenen ärztlichen Anordnung auch nutzen.

- Allerdings ist zu beachten, dass ein relevanter Anteil der Infektionen mit SARS-CoV-2 asymptomatisch verläuft, und auch bei den symptomatischen Fällen besteht eine hohe Infektiosität bereits 1 - 2 Tage vor Symptombeginn. Durch eine alleinige gezielte Anamnese vor Aufnahme in ein Schlaflabor kann daher kein sicherer Ausschluss eines erhöhten Infektionsrisikos durch eine SARS-CoV-2-Infektion erfolgen. Daher müssen alle im Schlaflabor geltenden Empfehlungen die Möglichkeit einer unbemerkten Untersuchung von SARS-CoV-2-infizierten Patienten mit einbeziehen.

\section{Polygrafie}

- Bei sachgerechter Desinfektion bzw. Austausch der Materialien ist ein sofortiger Wiedereinsatz möglich.

- Bei der materiellen Aufbereitung sind allgemeine (RKI) und von den Herstellern angegebenen Hygiene- und Desinfektionsstandards anzuwenden. 


\section{Therapie mit PAP im Schlaflabor/ Krankenhaus}

\section{Hintergrund}

Auch unter dem Einfluss der Corona-Pandemie sollte angestrebt werden, die Empfehlungen der S3-Leitlinie „Nicht erholsamer Schlaf/Schlafstörungen - Kapitel Schlafbezogene Atmungsstörungen “ [2] einzuhalten. Eine unkritische apparative Therapieeinleitung mit bspw. Verfahren der Überdrucktherapie ohne vorherige apparative Diagnostik alleine aufgrund der klinischen Einschätzung ist weiterhin abzulehnen.

Es gibt keine gesicherten Hinweise für eine erhöhte Empfänglichkeit oder Verschlechterung einer COVID-19 durch eine vorbestehende PAP-Therapie, vielmehr sind sogar positive Effekte beschrieben. Bei bestehender Infektion mit SARS-CoV-2 sollte eine vorbestehende Maskentherapie daher unter Einhaltung der beschriebenen Vorgaben zunächst fortgeführt werden, da eine Therapiebeendigung mit einer zusätzlichen kardiopulmonalen Belastung durch die unbehandelte Schlafapnoe einhergeht.

Bei Mitarbeitern, die Patienten mit nicht-invasiver Beatmung im Rahmen der SARS-Epidemie betreuten, wurde ein erhöhtes Infektionsrisiko beobachtet [23, 24], welches jedoch eher durch die nötige Nähe zum Patienten erklärt werden kann, da auch beim Schreiben eines EKGs ein in gleicher Weise erhöhtes Infektionsrisiko bestand. Es herrscht dennoch eine große Verunsicherung bzgl. einer unbemerkten Verbreitung von Corona-Viren durch vermehrte Aerosolbildung bei Anwendung einer Positivdrucktherapie (PAP, NIV) mit Verwendung verschiedener Maskensysteme $[25,26]$. Dieser Aspekt ist sehr detailliert im Positionspapier der DPG zur praktischen Umsetzung der apparativen Differenzialtherapie der akuten respiratorischen Insuffizienz bei COVID-19 vom 17.04.2020 mit ausführlichen entsprechenden Literaturhinweisen berücksichtigt [1]. Die dortigen Empfehlungen und Aussagen gelten größtenteils analog auch für die nächtliche Überdrucktherapie zur Behandlung schlafbezogener Atmungsstörungen.

Für die Schlafmedizin relevante grundlegende Aspekte sind hier kurz zusammengefasst:

- Nach vorherrschender Meinung erfolgt die Infektion mit SARS-CoV-2 vorwiegend über eine Tröpfcheninfektion. Eine Infektion ist aber auch durch Schwebepartikel möglich, bei Influenza werden sogar 50\% der Infektionen auf diesem Wege angenommen. Das SARS-CoV-2 hat einen variablen Durchmesser, der zwischen 60 und 140 Nanometern liegt.

- Jeder Mensch atmet Aerosole mit einem breiten Partikelspektrum ab, die Größe der abgeatmeten Partikel nimmt durch Verlust von Wasser rasch auf 0,3 mm ab. Die Zahl der abgeatmeten Partikel ist individuell unterschiedlich und schwankt zwischen 100 und 50000 pro Atemzug, sie steigt bei bronchialen Infekten deutlich an, sodass einzelne Menschen dann sehr viele Partikel abatmen (sog. Superspreader). Dies ist insofern von Bedeutung, als dass möglicherweise eine hohe Viruslast bei Erstinfektion mit einem schwereren Verlauf einhergehen kann [27, 28]. Das Volumen eines Aerosolpartikels errechnet sich aus der 3. Potenz des
Radius. Ein Partikel der Größe $10 \mu \mathrm{m}$ hat daher das Volumen von 1000 Partikeln der Größe 1 um [29]. Es konnte bei Influenza-Patienten gezeigt werden, dass virale RNA bereits bei normaler Ruheatmung abgegeben wird. Dabei lag die kumulative RNA-Menge in Partikeln $<5 \mu \mathrm{m}$ sogar etwas oberhalb der Menge, die aus Partikeln $>5 \mu \mathrm{m}$ isoliert werden konnte.

- Infektiöse respiratorische Aerosole entstehen auf Alveolarebene, sie folgen bis zu einer Partikelgröße von 3-4 mm dem Trägergas (hier Luft). Kleine Partikel (1 mm, d. h. 10× größer als das Virus) können für mindestens 3 Stunden in der Luft schweben [30]. Allerdings nimmt die Vitalität von Viren im Aerosol mit der Zeit ab, bei Influenzaviren um den Faktor $10^{2}$ nach 90 min [31], die Halbwertzeit des vitalen SARS CoV1- und -2-Virus in der Luft betrug 1,1 bis 1,2 Stunden [30].

- Bei symptomatischen und asymptomatischen Patienten fand sich die gleiche SARS-CoV-2-Viruslast in naso- und oropharyngealen Abstrichen. Daher kommt es wahrscheinlich nicht zu einer geringeren Virusverbreitung durch eine Positivdrucktherapie, wenn der Patient asymptomatisch ist [32].

- Grundsätzlich kann die Anwendung einer Positivdrucktherapie über verschiedene Maskensysteme mit der Bildung einer infektiösen Aerosolwolke einhergehen [1,33-36]. Ob sich durch den Einsatz von PAP oder NIV die Menge des abgeatmeten Virus bei einer bestehenden Infektion verändert, ist bisher nicht untersucht worden. Frühere Untersuchungen zeigten, dass unter NIV lediglich die Anzahl größerer Partikel (>10 mm) zunahm, und das auch nur bei Patienten mit Schnupfensymptomatik. Partikel dieser Größe sedimentieren schnell und sind daher für das direkte Umfeld des Patienten relevant [37].

- Faktoren, die das Risiko einer Ansteckung durch Aerosole bestimmen sind: Virusdichte im Aerosol, Halbwertszeit vitaler Viren im Aerosol, Reichweite der Aerosolwolke, Größe der Partikel, Raumgröße und Belüftung, Viruslast des infizierten Patienten (Low- oder Superspreader).

- Experimentelle Untersuchungen zeigen durch Verwendung verschiedener Leckageysteme bei den überwiegend in der Therapie der Schlafapnoe eingesetzten vented Masken eine rauchmarkierte Luftwolke bis zu einer Entfernung von $85 \mathrm{~cm}$, durch die Maskenleckage bei undichtem Sitz jedoch nur in $10 \mathrm{~cm}$ [38]. Neuere Publikationen konnten zeigen, dass unter den aktuellen Masken bei CPAP-Therapie nur eine sehr geringe Luftverwirbelung bis etwa $34 \mathrm{~cm}$ auftritt [38]. Zum Vergleich: Die maximale Aerosolwolke betrug in einer Analyse von Tang et al. bei normaler Atmung und Niesen $0,6 \mathrm{~m}$, beim Husten 0,8 m [39]. Neuere Untersuchungen mit Hochgeschwindigkeitskameras zeigten jedoch Reichweiten bis zu $8 \mathrm{~m}$ beim Niesen [40]. Bei Anwendung einer nasalen High-Flow-Therapie (NHF) verlängert sich die durch Rauchpartikel markierte Exspirationswolke lediglich von 6,5 auf $17,2 \mathrm{~cm}$ nach einer Erhöhung des nasalen Flusses von 10 auf 60 Liter pro Minute [41]. Im Vergleich zu einer Sauerstofftherapie über eine Sauerstoffmaske, die den Exspirationsstrom deutlich verringert, erhöhte die NHF die Keimabgabe bei bakterieller Pneumonie nicht [42]. Bemerkenswerter- 
weise vermindert eine isotone Kochsalzinhalation die Abgabe von Aerosolen aus der Lunge für bis zu 6 Stunden um durchschnittlich $72 \%$ [43]. Ursache ist wahrscheinlich eine veränderte Oberflächenspannung des Flüssigkeitsfilms auf Atemwegsepithelien. Hinweise für eine vermehrtes Infektionsrisiko des medizinischen Personals bei Anwendung von Verneblertherapien fanden sich nicht [24,44].

- Bei Anwendung einer Sauerstofftherapie über Venturi-Masken zeigte sich eine Exspirationswolke im Abstand von 20 $40 \mathrm{~cm}$ vor dem Gesicht bei Flussraten von 4-10 L Sauerstoff pro Minute. Bei Anwendung einer Sauerstofftherapie über eine Nasenbrille mit Flussraten von 1 bzw. $5 \mathrm{~L} / \mathrm{min}$ zeigte sich eine Explorationswolke mit einer Ausdehnung von $66 \mathrm{~cm}$ bzw. $1 \mathrm{~m} \mathrm{[38].}$

- Daten zur Veränderung der Aerosolwolke durch SauerstoffBeimengung bei Anwendung einer Maskenüberdrucktherapie sind nicht bekannt.

- Folgende Kernaussagen und Feststellungen aus dem DGPPositionspapier zur praktischen Umsetzung der apparativen Differenzialtherapie der akuten respiratorischen Insuffizienz bei COVID-19 [1] lassen sich auch auf die Therapie von Patienten im Schlaflabor übertragen:

Kernaussage 3.1: Offene Systeme bzw. Leckagesysteme (sog. vented Masken) können die Abgabe von respirablen Partikeln erhöhen, (ungewollte) Maskenleckagen spielen bei der Aerosolbildung eine eher untergeordnete Rolle.

Feststellung 3.2: Eine nasale High-Flow-Therapie (NHF) vergrößert die exspirierte Aerosolwolke um einige Zentimeter. Eine relevant vermehrte Abgabe infektiöser Aerosole im Vergleich zum spontan atmenden Patienten konnte weder in vitro noch in vivo bisher nachgewiesen werden.

Kernaussage 3.3: Mit persönlicher Schutzausrüstung (Augenschutz, FFP2- bzw. FFP3-Maske, Kittel) kann eine Inhalationstherapie, NHF-Therapie, eine PAP-Therapie oder eine NIV nach jetzigem Kenntnisstand vom Personal ohne erhöhtes Infektionsrisiko durchgeführt werden.

Feststellung 3.4: Die Sauerstoffgabe über Maske oder Nasensonden führt zu keiner vermehrten Aerosolbildung. Verschiedene Sauerstoffsysteme (Nasensonden, Sauerstoff-Masken und Venturi-Masken) können die Luft bei der Exspiration unterschiedlich ablenken, nur bei Nasensonden mit hohen Sauerstoffflüssen ist die bei der Ausatmung gebildete Aerosolwolke in ihrer Reichweite größer als unter Spontanatmung.

- Bei SARS-CoV-2-positiven Patienten empfiehlt die DPG zur apparativen Beatmungstherapie den Einsatz von virendichten Filtern vor dem Exspirationssystem der PAP/NIV-Geräte [1]. Anders als bei diesen Patienten dient die PAP/NIV-Therapie im Schlaflabor nicht der Therapie der akuten respiratorischen Insuffizienz, sondern der Therapie der schlafbezogenen Atmungsstörung bzw. der Hypoventilation. Gleichzeitig sollen die oben aufgeführten Screeningmaßnahmen dazu führen, SARS-CoV-2-positive Patienten im Vorfeld zu detektieren und erst nach der Erkrankung der elektiven
Therapie im Schlaflabor zuzuführen. Des Weiteren ist unklar, in wieweit der zusätzliche Widerstand des Virenfilters und die Verwendung von non-vented Masken die Regelalgorithmen (z. B. forcierte Oszillationstechnik, FOT) beeinträchtigen. Zusätzlich ist das Portfolio an non-vented Masken, insbesondere Nasenmasken im Vergleich zu den ventedSystemen deutlich eingeschränkt.

Wenn die Schlaflaborpatienten im Einzelzimmer liegen, die betreuenden Mitarbeiter der Schlaflabors strikt die geforderten Hygienemaßnahmen inkl. Nutzung einer FFP2/FFP3Maske einhalten und sie sich vom direkten Abstrom der an der Maske entweichenden Luft fernhalten, besteht nach physikalischen Gesichtspunkten kein erhöhtes Risiko einer Infektion verglichen, mit spontan atmenden Personen. Ob non-vented-Masken und/oder Virenfilter das Risiko vermindern, ist bisher nicht untersucht, eine Verminderung der Aerosolproduktion ist jedoch nachgewiesen [37].

Im Rahmen einer Risiko-Nutzen-Bewertung halten die Autoren dieser Stellungnahme daher zum jetzigen Zeitpunkt den ausschließlichen Einsatz von non-vented-Masken mit Virenfiltern bei elektiv betreuten Schlaflaborpatienten ohne konkrete Hinweise für eine SARS-CoV-2-Infektion für nicht gerechtfertigt.

\section{Konkrete Empfehlungen zur Überdrucktherapie im Zusammenhang mit der Corona-Pandemie}

- Patienten mit schlafbezogenen Atmungsstörungen sollten eine PAP-Therapie und andere Überdruckverfahren zuhause normal weiter nutzen.

- Bei fehlenden Hinweisen für eine SARS-CoV-2-Infektion (Abklärung s.o.) kann die Anpassung oder Kontrolle einer Überdrucktherapie (PAP/NIV) unter strikter Einhaltung der Hygienerichtlinien im Schlaflabor erfolgen.

- Sollte ein Schlafapnoe Patient mit PAP-Therapie symptomatisch werden hinsichtlich einer COVID-19, gelten die aktuellen Empfehlungen zu Diagnostik und Quarantäne (aktualisierte Version erhältlich über Hompage RKI).

- Bei notwendiger stationärer Aufnahme und bestätigter oder vermuteter SARS-CoV-2-Infektion kann außerhalb des Schlaflabores eine Überdrucktherapie (PAP, NIV) unter strikter Einhaltung der Hygienerichtlinien (Einzelzimmer, geeignete PSA inkl. FFP2/FFP3-Maske, Augenschutz) durchgeführt werden.

- Die Nutzung von Warmluftbefeuchtern kann auch bei gesicherter SARS-CoV-2-Infektion fortgeführt werden, da diese keine Aerosole erzeugen [45].

- Die allgemeinen Hinweise zu Pflege und Reinigung der Masken/Schläuche sowie Austausch der Filter gelten unverändert. Die meisten Hersteller haben Reinigungshinweise für PAP-Geräte, Masken und Diagnostikgeräte veröffentlicht.

- Selbstverständlich dürfen Masken, Schläuche und PAP-/ Beatmungsgeräte zuhause nur patientenbezogen genutzt und nicht ausgetauscht werden.

- Wir empfehlen einen behutsamen Umgang mit Maske, Schlauch und Gerät, da es zu vorübergehenden Lieferengpässen kommen kann. 
- Die o. g. Hinweise zu Klimaanlagen (s. o.) gelten auch bei häuslicher Quarantäne und Maskennutzung. Nicht genutzt werden sollte eine zentrale Klimaanlage mit Umluft zwischen mehreren Räumen, raumbezogene Klimaanlagen mit Abluft nach außen sind hingegen unproblematisch.

- Gemäß der RKI-Empfehlung sollte eine tägliche Wischdesinfektion der patientennahen (Handkontakt-)Flächen (z. B. Nachttisch, Nassbereich, Türgriffe) mit einem Flächendesinfektionsmittel mit nachgewiesener, mindestens begrenzt viruzider Wirksamkeit (siehe RKI-Liste geprüfter und anerkannter Desinfektionsmittel und -verfahren) erfolgen. Bei Bedarf sind die Desinfektionsmaßnahmen auf weitere kontaminationsgefährdete bzw. kontaminierte Flächen auszudehnen.

- Alle Medizinprodukte mit direktem Kontakt zum Patienten sind patientenbezogen zu verwenden und müssen nach Gebrauch desinfiziert werden.

\section{EMPFEHLUNGEN IN KURZFORM}

\section{Allgemeine Kommentare}

- Die Fortführung bzw. Wiederaufnahme schlafmedizinischer Leistungen unter den gebotenen Hygienemaßnahmen ist dringend anzustreben zur Vermeidung medizinischer und psychosozialer Komplikationen.

- Es gibt keine gesicherten Hinweise für eine Verschlechterung der COVID-19 durch eine PAP-Therapie.

- Grundsätzlich kann die Anwendung einer Positivdrucktherapie über verschiedene Maskensysteme mit der Bildung einer infektiösen Aerosolwolke einhergehen.

- Bei bestätigter Infektion mit SARS-CoV-2 sollte im ambulanten Umfeld eine vorbestehende PAP-Therapie unter Einhaltung der Vorgaben des RKI zur häuslichen Isolierung fortgeführt werden, da eine Therapiebeendigung mit einer zusätzlichen kardiopulmonalen Belastung durch die unbehandelte schlafbezogene Atmungsstörung einhergeht.

- Mit persönlicher Schutzausrüstung (Augenschutz, FFP2/ FFP3-Maske, Handschuhe, Schutzkittel/-anzug) kann eine Inhalationstherapie, NHF-Therapie, eine PAP-Therapie oder eine NIV nach jetzigem Kenntnisstand vom Personal ohne erhöhtes Infektionsrisiko durchgeführt werden.

- Viele Aspekte aus dem DGP-Positionspapier zur praktischen Umsetzung der apparativen Differenzialtherapie der akuten respiratorischen Insuffizienz bei COVID-19 [1] lassen sich auch auf schlafbezogene Atmungsstörungen übertragen, Abweichungen sind im Text kommentiert.

\section{Allgemeine Empfehlungen zur Organisation und Durchführung schlafmedizinischer Leistungen}

- Aktuelle bundes- und länderspezifischen Vorgaben beachten.

- Rücksprache mit dem Krankenhaushygieniker bzw. dem lokalen Gesundheitsamt notwendig bei Wiederauf- nahme des Schlaflaborbetriebes und relevanten organisatorischen Änderungen.

- Konsequente Einhaltung der auch in der Öffentlichkeit aktuell geltenden Abstandsregeln (1,5-2 m) und Maskenpflicht (Ausnahme: während Polysomnografie, Polygrafie und PAP-Therapie).

- Unter Berücksichtigung der räumlichen Gegebenheiten konsequente räumliche Abtrennung des Schlaflabores, sofern im oder am Krankenhaus befindlich, in eine „Non-COVID-Zone“ mit Vermeidung von Patientenkontakt durch getrennte Zugänge und Wartebereiche.

- Vor Aufnahme sollte eine angemessene Evaluation hinsichtlich des Gesundheitszustandes und des Infektionsund Erkrankungsrisikos der Patienten erfolgen, z. B. 1 -2 Tage vor Aufnahme (telefonische) Abfrage bzgl. des Gesundheitszustandes und möglicher SARS-CoV-2Kontakte.

Bei Hinweisen für eine SARS-CoV-2-Infektion bzw. COVID-19 Verschiebung des Termins.

Bei Aufnahme nochmalige Abfrage bezüglich typischer COVID-19-Symptome und möglicher SARS-CoV-2-Kontakte (entsprechende Dokumentation dringend angeraten).

- Vermeidung unnötiger Kontakte z. B. durch zeitlich gestaffelte Einbestellung sowie ggf. Verringerung der Kontaktzeiten durch abendliche Aufnahme.

- Die in der aktuellen S3-Leitlinie „Nicht erholsamer Schlaf/Schlafstörungen - Kapitel Schlafbezogene Atmungsstörungen“ ([2] derzeit in Überarbeitung) empfohlenen Grundlagen zur Durchführung und Überwachung einer schlafmedizinischen Diagnostik und Therapieeinleitung sind auch unter dem Einfluss der Corona-Pandemie zu berücksichtigen. Eine Leistungserbringung außerhalb dieser Vorgaben ist auch mit Verweis auf die Pandemie aus qualitativen und abrechnungstechnischen Aspekten abzulehnen. Dies gilt ausdrücklich auch für Geräteanpassung und Maskenanpassung durch die Provider.

\section{Priorisierung}

- Zum ersten Patientenkontakt, falls vorhanden, ggf. Nutzung telefonischer/videotelefonischer Vorabberatungen mit Einstufung der Notwendigkeit einer persönlichen Vorstellung zur Diagnostik, Therapieeinleitung oder-kontrolle.

- Bei unzureichenden Schlaflaborressourcen Priorisierung bzgl. der Dringlichkeit (kardiovaskuläre und andere Komorbiditäten, Risikoberufe, Leidensdruck, soziale Faktoren wie Erhalt der Erwerbsfähigkeit bei Berufskraftfahrern etc.).

- Bei zurückgestellter Diagnostik, Therapieeinleitung oder -kontrolle eines unter Therapie noch symptomatischen Patienten sollte eine Aufklärung bzgl. der resultierenden Risiken inkl. einer ggf. nicht vorliegenden medizinischen Fahrtauglichkeit erfolgen. 


\section{Infektprävention im Schlaflabor}

- Strikte Beachtung der Basishygiene mit geeigneter persönlicher Schutzausrüstung (PSA, bei normalem Patientenkontakt inkl. Mund-Nasenschutz [MNS], bei engem Kontakt durch Verkabelung und Maskenanpassung/-optimierung inkl. Augenschutz und FFP2/FFP3Maske).

- Unterbringung im Einzelzimmer vorzugsweise mit eigener Nasszelle.

- Kein gemeinsamer Aufenthalt in Aufenthaltsräumen, auch sonst Vermeidung eines Kontaktes der Patienten untereinander.

- Besucherregelungen unter Berücksichtigung aktueller lokaler Vorgaben.

- Sofern verfügbar bevorzugt Einmalmaterial, ansonsten strikte Einhaltung der RKI- bzw. Herstellerempfehlungen zur Desinfektion und Hygiene von Oberflächen, Kabeln, PAP- und Beatmungsgeräten, Masken, Schläuchen, Filtern.

- Gute Durchlüftung der Räume nach außen. Keine Nutzung zentraler Klimaanlagen mit Umluft zwischen mehreren Räumen. Raumbezogene Klimaanlagen mit Abluft nach außen sind unproblematisch.

- Kontaktzeiten im Untersuchungsraum möglichst kurz halten, z. B. durch die bevorzugte Nutzung der Sprechanlage oder spezielle Besprechungsräume mit erweiterten Schutzmaßnahmen (Abstand, gegebenenfalls Plexiglasscheibe).

\section{Screening und Schutz von Patienten und Personal}

- Bzgl. der Kriterien für eine Testung von Schlaflaborpatienten auf SARS-CoV-2-Infektion gelten die aktuellen Empfehlungen des RKI bzw. der Bundes- oder Landesbehörden. Zum Zeitpunkt der Manuskripterstellung gilt: Bei akuten respiratorischen Symptomen jeder Schwere und/oder Geruchs-/Geschmacksstörungen, SARS-CoV2-Kontakt innerhalb von 14 Tagen oder klinischen bzw. radiologischen Hinweisen auf eine virale Pneumonie sollte eine Testung auf SARS-CoV-2 durchgeführt werden (COVID-19-Verdacht: Testkriterien und Maßnahmen. Orientierungshilfe für Ärztinnen und Ärzte, Version 12. Mai 2020, bitte Aktualisierung beachten (RKI - Allgemeine Quellen). Dann erfolgt keine Untersuchung im Schlaflabor.

- Bei Auftreten typischer Symptome oder bestehendem Kontakt mit SARS-CoV-2-Infizierten während des Aufenthaltes im Schlaflabor Abklärung der Patienten und des medizinischen Personals gemäß der aktuellen Empfehlung des RKI (s. o.).

- Bei Symptomen oder sonstigem Verdacht auf COVID-19 (typisches Röntgen, SARS-CoV-2-Kontakt) und notwendiger stationärer Aufnahme außerhalb des Schlaflabores sollten OSA-Patienten ihr PAP-Gerät mitbringen und gemäß einer auf ihren aktuellen Gesundheitszustand bezogenen ärztlichen Anordnung nutzen.
- Ein relevanter Anteil der Infektionen mit SARS-CoV-2 verläuft asymptomatisch, und auch bei den symptomatischen Fällen besteht eine hohe Infektiosität bereits 1 - 2 Tage vor Symptombeginn. Durch eine alleinige gezielte Anamnese vor Aufnahme kann somit kein Ausschluss eines erhöhten Infektionsrisikos durch eine SARS-CoV-2-Infektion im Schlaflabor erfolgen. Daher müssen alle im Schlaflabor geltenden Empfehlungen die Möglichkeit einer Untersuchung von SARS-CoV-2infizierten Patienten mit einbeziehen.

\section{Polygrafie}

- Bei sachgerechter Desinfektion bzw. Austausch der Materialien sofortiger Wiedereinsatz möglich. Gerät muss nicht in Quarantäne.

- Bei der materiellen Aufbereitung allgemeine (RKI) und von den Herstellern angegebene Hygiene- und Desinfektionsstandards beachten.

\section{Konkrete Empfehlungen zur Überdrucktherapie im Zusammenhang mit der Corona-Pandemie}

- Bei fehlenden Hinweisen für eine SARS-CoV-2-Infektion (Abklärung s. o.) ist eine Anpassung oder Kontrolle einer Überdrucktherapie (PAP/NIV) unter strikter Einhaltung der Hygienerichtlinien im Schlaflabor möglich.

- Bei notwendiger stationärer Aufnahme und bestätigter oder vermuteter SARS-CoV-2-Infektion kann außerhalb des Schlaflabores eine Positivdrucktherapie (PAP, NIV) unter strikter Einhaltung der Hygienerichtlinien (Einzelzimmer, geeignete PSA inkl. FFP2/FFP3-Maske, Augenschutz) durchgeführt werden.

- Die Nutzung von Warmluftbefeuchtern ist auch bei gesicherter SARS-CoV-2-Infektion weiter möglich, da diese keine Aerosole erzeugen.

- Allgemeine Hinweise zu Pflege und Reinigung der Masken/Schläuche sowie Austausch der Filter gelten unverändert. Herstellerangaben beachten.

- Behutsamer Umgang mit Maske, Schlauch und Gerät, da es zu vorübergehenden Lieferengpässen kommen kann.

- Gemäß RKI-Empfehlung tägliche Wischdesinfektion der patientennahen (Handkontakt-)Flächen (z. B. Nachttisch, Nassbereich, Türgriffe) mit einem Flächendesinfektionsmittel mit nachgewiesener, mindestens begrenzt viruzider Wirksamkeit (siehe RKI-Liste geprüfter und anerkannter Desinfektionsmittel und -verfahren). Bei Bedarf Desinfektionsmaßnahmen auf weitere kontaminationsgefährdete bzw. kontaminierte Flächen ausdehnen.

- Alle Medizinprodukte mit direktem Kontakt zum Patienten patientenbezogen verwenden und nach Gebrauch desinfizieren. 
ZUSAMMENFASSENDE EMPFEHLUNGEN FÜR DIE DIAGNOSTIK UND THERAPIE SCHLAFBEZOGENER ATMUNGSSTÖRUNGEN IM ZUSAMMENHANG MIT DER CORONA-PANDEMIE

- Die Fortführung bzw. Wiederaufnahme schlafmedizinischer Leistungen unter den gebotenen Hygienemaßnahmen ist dringend anzustreben zur Vermeidung medizinischer und psychosozialer Komplikationen.

- Es gibt keine gesicherten Hinweise für eine Verschlechterung der COVID-19 durch eine CPAP-Therapie.

- Grundsätzlich kann die Anwendung einer Überdrucktherapie über verschiedene Maskensysteme mit der Bildung einer infektiösen Aerosolwolke einhergehen. Die Aerosolwolke durch Masken mit verschiedenen Leckagesysthemen erreicht $0,85 \mathrm{~m}$ und damit etwa die Entfernung, die beim Husten erreicht wird. Maskenleckagen durch undichten Sitz verursachen eine Aerosolwolke von $10 \mathrm{~cm}$ Reichweite.

- Bei bestätigter Infektion mit SARS-CoV-2 sollte im ambulanten Umfeld eine vorbestehende Maskentherapie unter Einhaltung der Vorgaben des RKI zur häuslichen Isolierung fortgeführt werden, da eine Therapiebeendigung mit einer zusätzlichen kardiopulmonalen Belastung durch die unbehandelte schlafbezogene Atmungsstörung einhergeht.

- Mit persönlicher Schutzausrüstung (Augenschutz, FFP2/ FFP-3-Maske, Handschuhe, Schutzkittel/-anzug) kann eine Inhalationstherapie, NHF-Therapie, eine PAP-Therapie oder eine NIV nach jetzigem Kenntnisstand vom Personal ohne erhöhtes Infektionsrisiko durchgeführt werden (entsprechend Kernaussage 3.3 aus dem DGPPositionspapier zur praktischen Umsetzung der apparativen Differenzialtherapie der akuten respiratorischen Insuffizienz bei COVID-19 [1].

- Warmluftbefeuchter können auch bei bestehender COVID-19 weiter eingesetzt werden.

- Bezüglich der Reinigung, Pflege, Desinfektion und Wiederaufbereitung der eingesetzten Geräte, Masken und Schläuche gelten die allgemeinen Hersteller-Empfehlungen.

\section{Allgemeine Quellen (Auswahl,} bitte Aktualisierung beachten!)

- Empfehlungen des Robert-Koch-Institutes zur SARAS-CoV2Infektion:

https://www.rki.de/DE/Content/InfAZ/N/Neuartiges_Coronavirus/nCoV_node.html

- Guidance regarding coronavirus (COVID-19) and Obstructive Sleep Apnoea (OSA): for people who routinely use continuous positive airway pressure (CPAP), their families and health care workers, 20 March 2020, Version 1.0 20/3/2020. Complied by Dr Sonya Craig and Dr Sophie West with the OSA Alliance (incorporating British Thoracic Society, British Sleep Society, Association for Respiratory Technology and
Physiology, Sleep Apnoea Trust Association):

https://brit-thoracic.org.uk/media/455098/osa-alliance-

cpap-covid-19-advice-20-3-20-v10.pdf

- ATS Patientenratgeber:

https://www.thoracic.org/patients/patient-resources/resources/coronavirus-patient.pdf

- World Health Organization (WHO): https://www.who.int/health-topics/coronavirus\#tab=tab_1

- Johns Hopkins University COVID-19 Dashboard: https://coronavirus.jhu.edu/map.html

- COVID-19: FAQs for Sleep Clinicians The American Academy of Sleep Medicine (AASM) Public Safety Committee is responding to frequently asked questions (FAQs) to help sleep clinicians and accredited sleep facilities address the spread of the novel coronavirus (COVID-19).

https://aasm.org/covid-19-resources/covid-19-faq/

- The American Academy of Sleep Medicine (AASM) COVID-19 Mitigation Strategies for Sleep Clinics and Labs - Updated, Updated April 8, 2020, Based on the mitigation strategies recommended by the Center for Disease Control and prevention (CDC).

http://www.cpsa.ca/wp-content/uploads/2020/04/AASMcovid-19-mitigation-strategies-sleep-clinics-labs-updated. pdf

- British Thoracic Society: Advice for those seeing patients with obstructive sleep apnoea.

https://brit-thoracic.org.uk/about-us/covid-19-information-for-the-respiratory-community/

- NHS. Guidance for the role and use of non-invasive respiratory support in adult patients with COVID-19 (confirmed or suspected). 6 April 2020, Version 3.

https://www.england.nhs.uk/coronavirus/wp-content/uploads/sites/52/2020/03/specialty-guide-NIV-respiratory-support-and-coronavirus-v3.pdf

\section{Interessenkonflikt}

H. Woehrle hat Vortrags- und Beratungshonorare von folgenden Firmen erhalten: Novartis, Astra Zeneca, ResMed, Inspire, medinfo, wikonect, Universitätsklinik Regensburg, Klinikum Nürnberg, BDI. D. Dellweg hat Vortragshonorare von Löwenstein Medical und Lungpacer erhalten. A. Wiater hat Beraterhonorare von der Firma Infectopharm erhalten. P. Young hat Vortraghonorare erhalten von SanofiGenzyme, UCB Pharma, Medice, Vanda, Löwenstein-Medical und Biogen. W. Randerath hat Honorare für Mitarbeit in einem Advisory Board der Firmen Philipps Respironics, SoClean, Bioprojet, Bayer Sleep Research sowie Honorar und Reisekosten für Vortragstätigkeiten der Firmen Boehringer Ingelheim, Heinen und Löwenstein, Vanda Pharma und Night Balance erhalten. Für Forschungsvorhaben des Wissenschaftlichen Instituts Bethanien hat er Forschungsgelder der Firmen Somnics, Philipps Respironics und Heinen und Löwenstein erhalten. N. Büchner, $\mathrm{H}$. Hein geben an, dass kein Interessenkonflikt besteht. 
Literatur

[1] Pfeifer M, Ewig S, Voshaar T et al. Position Paper for the State of the Art Application of Respiratory Support in Patients with COVID-19 German Respiratory Society. Pneumologie 2020. doi:10.1055/a1157-9976

[2] Mayer G, Arzt M, Braumann B et al. S3-Leitlinie Nicht erholsamer Schlaf/Schlafstörungen - Kapitel „Schlafbezogene Atmungsstörungen“. Somnologie 2017; 20: 97-180

[3] CDC CfDCaP. Coronavirus Disease 2019 (COVID-19) - Transmission. In: Centers for Disease Control and Prevention; 2020

[4] Wu YC, Chen CS, Chan Y]. The outbreak of COVID-19: An overview. J Chin Med Assoc 2020; 83: 217-220

[5] WHO. World Health Organization: Coronavirus disease 2019 (COVID19) Situation Report - 87. 2020 (last accessed on 12 May 2020)

[6] He X, Lau EHY, Wu P et al. Temporal dynamics in viral shedding and transmissibility of COVID-19. Nat Med 2020. doi:10.1038/s41591020-0869-5

[7] Ganyani T, Kremer C, Chen D et al. Estimating the generation interval for COVID-19 based on symptom onset data. medRxiv: The Preprint Server for Health Sciences 2020. doi:10.1101/2020.03.05.20031815

[8] Kucirka L, Lauer S, Laeyendecker O et al. Variation in False-Negative Rate of Reverse Transcriptase Polymerase Chain Reaction-Based SARS-CoV-2 Tests by Time Since Exposure. Annals of Internal Medicine 2020. doi:10.7326/m20-1495

[9] Lavezzo E, Franchin E, Ciavarella C et al. Suppression of COVID-19 outbreak in the municipality of Vo. medRxiv: The Preprint Server for Health Sciences 2020. doi:10.1101/2020.04.17.20053157

[10] Streeck H, Schulte B, Kümmerer B et al. Infection fatality rate of SARSCoV-2 infection in a German community with a super-spreading event. medRxiv: The Preprint Server for Health Sciences 2020. doi: $10.1101 / 2020.05 .04 .20090076$

[11] Nishiura H, Kobayashi T, Miyama T et al. Estimation of the asymptomatic ratio of novel coronavirus infections (COVID-19). Int J Infect Dis 2020; 94: 154-155

[12] Fauci AS, Lane HC, Redfield RR. Covid-19 - Navigating the Uncharted. N Engl J Med 2020; 382: 1268-1269

[13] Rajgor DD, Lee MH, Archuleta $S$ et al. The many estimates of the COVID-19 case fatality rate. Lancet Infect Dis 2020. doi:10.1016/S14733099(20)30244-9

[14] Salje H, Tran Kiem C, Lefrancq N et al. Estimating the burden of SARS-CoV-2 in France. 2020. pasteur-02548181

[15] Bendavid E, Mulaney B, Sood N et al. COVID-19 Antibody Seroprevalence in Santa Clara County, California. medRxiv 2020. doi:10.1101/ 2020.04.14.20062463

[16] Gianicolo ERN, Blettner M, Karch A. Epidemiological measures in the context of the COVID-19 pandemic. Dtsch Arztebl Int 2020; 117: 336-342

[17] Chen T, Wu D, Chen H et al. Clinical characteristics of 113 deceased patients with coronavirus disease 2019: retrospective study. BM] 2020; 368: m1091

[18] Dreher MKA, Bickenbach J, Balfanz P et al. The characteristics of 50 hospitalized COVID-19 patients with and without ARDS. Dtsch ArztebI 2020; 117: 271-278

[19] Grote L, McNicholas WT, Hedner J et al. Sleep apnoea management in Europe during the COVID-19 pandemic: data from the European Sleep Apnoea Database (ESADA). Eur Respir J 2020. doi:10.1183/ 13993003.01323-2020

[20] Wang J, Yu W, Gao M et al. Impact of Obstructive Sleep Apnea Syndrome on Endothelial Function, Arterial Stiffening, and Serum Inflammatory Markers: An Updated Meta-analysis and Metaregression of 18 Studies. J Am Heart Assoc 2015; 4: e002454
[21] McSharry D, Malhotra A. Potential influences of obstructive sleep apnea and obesity on COVID-19 severity. J Clin Sleep Med 2020. doi: $10.5664 /$ jcsm. 8538

[22] Schwarz El, Schlatzer C, Rossi VA et al. Effect of CPAP Withdrawal on BP in OSA: Data from Three Randomized Controlled Trials. Chest 2016; 150: 1202-1210

[23] Fowler RA, Guest CB, Lapinsky SE et al. Transmission of severe acute respiratory syndrome during intubation and mechanical ventilation. Am J Respir Crit Care Med 2004; 169: 1198-1202

[24] Raboud JSA, McGeer A et al. Risk factors for SARS transmission from patients requiring intubation: a multicentre investigation in Toronto, Canada. PLoS One 2010; 5: e10717

[25] Baker JGSM. Controversies and challenges in respiratory medicine: Case for continuing community NIV and CPAP during the COVID-19 epidemic. Thorax 2020; 75: 368

[26] Barker JOO, Koeckerling D et al. COVID-19: community CPAP and NIV should be stopped unless medically necessary to support life. Thorax 2020; 75: 367

[27] Chu CM, Poon LL, Cheng VC et al. Initial viral load and the outcomes of SARS. CMAJ 2004; 171: 1349-1352

[28] Liu Y, Yan LM, Wan L et al. Viral dynamics in mild and severe cases of COVID-19. Lancet Infect Dis 2020. doi:10.1016/S1473-3099(20) 30232-2

[29] Köhler D, Fleischer W. Inhalationstherapie. München: Arcis Verlag; 2000

[30] van Doremalen N, Bushmaker T, Morris DH et al. Aerosol and Surface Stability of SARS-CoV-2 as Compared with SARS-CoV-1. N Engl J Med 2020; 382: 1564-1567

[31] Brown JR, Tang JW, Pankhurst L et al. Influenza virus survival in aerosols and estimates of viable virus loss resulting from aerosolization and air-sampling. J Hosp Infect 2015; 91: 278-281

[32] Zou L, Ruan F, Huang M et al. SARS-CoV-2 Viral Load in Upper Respiratory Specimens of Infected Patients. N Engl J Med 2020; 382: 11771179

[33] Brewster D], Chrimes N, Do TB et al. Consensus statement: Safe Airway Society principles of airway management and tracheal intubation specific to the COVID-19 adult patient group. Med J Aust 2020. doi: $10.5694 / \mathrm{mja} 2.50598$

[34] Tang JW, Li Y, Eames I et al. Factors involved in the aerosol transmission of infection and control of ventilation in healthcare premises. J Hosp Infect 2006; 64: 100-114

[35] WHO. Rational use of personal protective equipment for coronavirus disease (COVID-19) and considerations during severe shortages. Interim guidance. 6. April 2020

[36] Tran K, Cimon K, Severn M et al. Aerosol generating procedures and risk of transmission of acute respiratory infections to healthcare workers: a systematic review. PLoS One 2012; 7: e35797

[37] Simonds AK, Hanak A, Chatwin M et al. Evaluation of droplet dispersion during non-invasive ventilation, oxygen therapy, nebuliser treatment and chest physiotherapy in clinical practice: implications for management of pandemic influenza and other airborne infections. Health Technol Assess 2010; 14: 131-172

[38] Hui DS, Chow BK, Ng SS et al. Exhaled air dispersion distances during noninvasive ventilation via different Respironics face masks. Chest 2009; 136: 998-1005

[39] Tang JW, Nicolle AD, Klettner CA et al. Airflow dynamics of human jets: sneezing and breathing - potential sources of infectious aerosols. PLoS One 2013; 8: e59970

[40] Bourouiba L. Turbulent Gas Clouds and Respiratory Pathogen Emissions: Potential Implications for Reducing Transmission of COVID-19. JAMA 2020. doi:10.1001/jama.2020.4756 
[41] Hui DS, Chow BK, Lo T et al. Exhaled air dispersion during high-flow nasal cannula therapy versus CPAP via different masks. Eur Respir J 2019; 53: 1802339

[42] Leung CCH, Joynt GM, Gomersall CD et al. Comparison of high-flow nasal cannula versus oxygen face mask for environmental bacterial contamination in critically ill pneumonia patients: a randomized controlled crossover trial. J Hosp Infect 2019; 101: 84-87
[43] Edwards DA, Man JC, Brand P et al. Inhaling to mitigate exhaled bioaerosols. Proc Natl Acad Sci U S A 2004; 101: 17383-17388

[44] Loeb M, McGeer A, Henry B et al. SARS among critical care nurses, Toronto. Emerg Infect Dis 2004; 10: 251-255

[45] Wenzel M, Klauke M, Gessenhardt F et al. Sterile water is unnecessary in a continuous positive airway pressure convection-type humidifier in the treatment of obstructive sleep apnea syndrome. Chest 2005; 128: $2138-2140$ 